\title{
Biogenesis pathways of piRNAs loaded onto AGO3 in the Drosophila testis
}

\author{
AKIHIRO NAGAO,,${ }^{1,2}$ TOUTAI MITUYAMA, ${ }^{3}$ HAIDONG HUANG,${ }^{4}$ DAHUA CHEN, ${ }^{4}$ MIKIKO C. SIOMI, ${ }^{1,5}$ \\ and HARUHIKO SIOMI \\ ${ }^{1}$ Department of Molecular Biology, Keio University School of Medicine, Tokyo 160-8582, Japan \\ ${ }^{2}$ Institute of Health Biosciences, University of Tokushima, Tokushima 770-8503, Japan \\ ${ }^{3}$ Computational Biology Research Center (CBRC), National Institute of Advanced Industrial Science and Technology (AIST), Tokyo 135-0064, \\ Japan \\ ${ }^{4}$ Institute of Zoology, Chinese Academy of Sciences, Beijing, People's Republic of China \\ ${ }^{5}$ JST, CREST, Saitama, Japan
}

\begin{abstract}
PIWI-interacting RNAs (piRNAs) silence transposable elements in animal germ cells. In Drosophila ovaries, piRNAs are produced by two distinct pathways: the "ping-pong" amplification cycle that operates in germ cells and a ping-pongindependent pathway termed the primary pathway that mainly operates in somatic cells. AGO3, one of three PIWI proteins in flies, is involved in the ping-pong cycle in ovaries. We characterized AGO3-associated piRNAs in fly testes and found that like in ovaries, AGO3 functions in the ping-pong cycle with Aubergine (Aub) for piRNA production from transposon transcripts. In contrast, most AGO3-associated piRNAs corresponding to Suppressor of Stellate [Su(Ste)] genes are antisense-oriented and bound to Aub. In addition, the vast majority of AGO3-bound piRNAs derived from the AT-chX locus on chromosome $X$ are antisense-oriented and are also found among Aub-associated piRNAs. The presence of very few sense $S u(S t e)$ and $A T$-chX piRNAs suggests that biogenesis of both $S u(S t e)$ and $A T$-chX piRNAs by a ping-pong mechanism only is highly unlikely. Nevertheless, the mutual interdependence of AGO3 and Aub for the accumulation of these piRNAs shows that their production relies on both AGO3 and Aub. Analysis of piRNA pathway mutants revealed that although the requirements for piRNA factors for $S u(S t e)$ - and $A T$-chX-piRNA levels mostly overlap and resemble those for the ping-pong mechanism in the ovaries, Armitage (armi) is not required for the accumulation of $A T$-chX-1 piRNA. These findings suggest that the impacts of armi mutants on the operation of the piRNA pathway are variable in germ cells of fly testes.
\end{abstract}

Keywords: AGO3; Aubergine; piRNA; Drosophila; RNA silencing; germline

\section{INTRODUCTION}

Recent studies have shown that eukaryotic cells express a large number of different small RNAs, 20- to 30-nucleotide (nt) long, which trigger various forms of sequence-specific gene silencing by guiding the Argonaute complex to target RNAs by base-pairing (Ghildiyal and Zamore 2009; Kim et al. 2009; Siomi and Siomi 2009). This process is referred to as "RNA silencing." RNA silencing is an evolutionarily conserved nucleic acid-based immunity that restrains the expression of parasitic and pathogenic invaders such as vi-

Reprint requests to: Haruhiko Siomi, Department of Molecular Biology, Keio University School of Medicine, Tokyo 160-8582, Japan; e-mail: awa403@sc.itc.keio.ac.jp; fax: 81-3-5363-3266.

Article published online ahead of print. Article and publication date are at http://www.rnajournal.org/cgi/doi/10.1261/rna.2270710. ruses and transposable elements (Girard and Hannon 2008; Siomi and Siomi 2008).

In Drosophila, the endogenous small interfering RNA (endo-siRNA or esiRNA) pathway restrains the expression of transposable elements in somatic cells, whereas the PIWI-interacting RNA (piRNA) pathway represses them in germline cells (Ghildiyal and Zamore 2009; Kim et al. 2009; Siomi and Siomi 2009). esiRNAs are produced by the Dicer2-dependent pathway, indicating that they are derived from double-stranded RNA (dsRNA) precursors (Czech et al. 2008; Ghildiyal et al. 2008; Kawamura et al. 2008; Okamura et al. 2008). Processed esiRNAs are loaded onto AGO2 to form RNA-induced silencing complexes (RISCs) that silence transposable elements by cleaving their transcripts. In contrast, the production of piRNAs is Dicerindependent, indicating that the biogenesis of piRNAs is distinct from that of esiRNAs and does not involve stable dsRNA intermediates (Vagin et al. 2006; Houwing et al. 
2007). In fly gonads, three distinct PIWI proteins of the Argonaute family_AGO3, Aubergine (Aub), and Piwi-are expressed (Williams and Rubin 2002). In fly ovaries, piRNAs associated with Aub and Piwi are derived mainly from the antisense strand of retrotransposons, while AGO3-associated piRNAs arise mainly from the sense strand. Aub- and Piwiassociated piRNAs show a strong preference for uracil (U) at their 5' ends, while AGO3-associated piRNAs show a preference for adenine $(\mathrm{A})$ at the tenth nucleotide from the $5^{\prime}$ end (Brennecke et al. 2007; Gunawardane et al. 2007). The first 10 nt of Aub-associated piRNAs can be complementary to the first $10 \mathrm{nt}$ of AGO3-associated piRNAs. These PIWI proteins retain the endoribonuclease or Slicer activity that allows them to cleave an RNA substrate across from position 10 of their bound piRNA (Saito et al. 2006; Gunawardane et al. 2007). These observations suggest a Slicer-dependent selfamplifying loop model, called the "ping-pong cycle," for piRNA biogenesis in which sense piRNAs bound to AGO3 cleave long antisense transcripts and guide the formation of the 5' end of the antisense piRNA bound to Aub, and vice versa (Brennecke et al. 2007; Gunawardane et al. 2007). Therefore, in this amplification loop, transposons are both a source gene of piRNAs and a target of piRNA-mediated silencing. Signatures of this ping-pong cycle are conserved among metazoans (Aravin et al. 2007; Houwing et al. 2007; Grimson et al. 2008; Kawaoka et al. 2009).

Both AGO3 and Aub are cytoplasmic proteins expressed only in germline cells in the ovaries (Harris and Macdonald 2001; Brennecke et al. 2007; Gunawardane et al. 2007; Nishida et al. 2007). In contrast, Piwi is nuclear and is expressed in both germline cells and somatic support cells such as follicle cells in the ovaries (Cox et al. 2000; Megosh et al. 2006; Saito et al. 2006; Brennecke et al. 2007; Gunawardane et al. 2007; Nishida et al. 2007). Piwi is therefore spatially separated from AGO3 and Aub at the cell-type and subcellular levels in the ovaries. The pingpong cycle in fly ovaries operates specifically in germ cells and engages mainly AGO3 and Aub (Brennecke et al. 2007; Gunawardane et al. 2007; Li et al. 2009; Malone et al. 2009). piRNAs produced by the ping-pong cycle are often referred to as secondary piRNAs. Classification of piRNAs according to their origins has indicated that piRNAs derived from a particular piRNA cluster locus, the flamenco locus, are exclusively loaded onto Piwi and not further amplified (Brennecke et al. 2007; Malone et al. 2009; Saito et al. 2009). These piRNAs are overwhelmingly antisense. The flamenco locus was originally identified as a repressor of transposon expression in somatic follicle cells (Pelisson et al. 1994), where Piwi, but not AGO3 and Aub, is expressed. These results indicate that piRNAs from the flamenco locus are produced by a pathway independent of the ping-pong cycle in ovarian somatic cells. This pathway is called the "primary piRNA pathway" (Brennecke et al. 2007; Malone et al. 2009; Saito et al. 2009; Siomi and KuramochiMiyagawa 2009). It is believed that the primary pathway also generates piRNAs that can initiate the ping-pong cycle in the ovarian germ cells (Brennecke et al. 2007; Malone et al. 2009). Although Aub receives some piRNAs via a primary biogenesis pathway operating in the ovarian germ cells, AGO3 contains mostly secondary piRNAs (Brennecke et al. 2007; Li et al. 2009; Malone et al. 2009).

In Drosophila testes, the X-linked Stellate locus is silenced by piRNAs derived from antisense transcripts of the homologous Suppressor of Stellate $[\mathrm{Su}(\mathrm{Ste})]$ repeats on chromosome Y (Balakireva et al. 1992; Bozzetti et al. 1995; Aravin et al. 2001, 2004; Vagin et al. 2006). Aub is required for accumulation of $\mathrm{Su}$ (Ste) piRNAs (Aravin et al. 2004; Vagin et al. 2006). Mutations in $A u b$ result in the formation of Stellate protein crystals in primary spermatocytes, which causes male sterility (Bozzetti et al. 1995; Aravin et al. 2001, 2004; Kotelnikov et al. 2009). We previously demonstrated that, among piRNAs associated with Aub in fly testes, those derived from $\mathrm{Su}(\mathrm{Ste})$ antisense transcripts were the most abundant (Nishida et al. 2007). The second largest class of piRNAs associated with Aub in the testes is derived from a repetitive region on chromosome $\mathrm{X}$, termed $A T$-chX. One of these piRNAs, termed $A T-c h X-1$, shows strong complementarity to vasa (vas) mRNA, a germline-specific transcript involved in oocyte differentiation and cyst development (Lasko and Ashburner 1988; Styhler et al. 1998). The $A T-c h X-1$ piRNA down-regulates the protein levels of VAS (Nishida et al. 2007; Li et al. 2009). piRNAs from the two loci, $\mathrm{Su}$ (Ste) and $A T-\operatorname{chX}$, are not bound to Piwi (Nishida et al. 2007). Recently, Li et al. (2009) produced Ago3 mutants and demonstrated that Ago3 is required for accumulation of both $S u(S t e)$ and $A T-c h X$ piRNAs in fly testes. However, how piRNAs are produced in fly testes remains largely unknown.

We sought to determine whether these abundant piRNAs in fly testes were produced in a ping-pong-dependent manner, as is the case for piRNAs derived from transposons in ovaries. Here, we analyzed piRNAs associated with AGO3 and Aub immunopurified from fly testes. Our data provide support for the ping-pong cycle in which transposonderived piRNAs are amplified by AGO3 and Aub in fly testes. However, a large number of piRNAs with exactly the same sequences, derived from antisense strands of the two loci, Su(Ste) and $A T-c h X$, were associated with both AGO3 and Aub. Therefore, biogenesis of these piRNAs through a ping-pong mechanism only is highly unlikely. We examined the accumulation of $S u(S t e)$ and $A T-\operatorname{chX}$ piRNAs in mutant testes defective for nine piRNA pathway proteins. We found that AGO3, Aub, spindle-E (Spn-E), Krimper (Krimp), Maelstrom (Mael), and VAS are required for the production of both types of piRNAs. However, the production of $S u(S t e)$ piRNAs, but not $A T-\operatorname{chX}-1$ piRNA, depends on the RNA helicase Armitage (Armi). Together, these results suggest that distinct piRNA pathways, with different genetic requirements probably depending on the piRNA loci, operate in germ cells of fly testes. 


\section{RESULTS}

\section{Expression of AGO3 in fly testes}

To biochemically investigate piRNA biogenesis in fly testes, we produced antibodies against AGO3 (Nishida et al. 2009). Western blotting of testis lysates prepared from yellow white wild-type (WT), trans-heterozygous ago3 mutants $\left(a g o 3^{t 2} / \operatorname{ago~}^{t 3}\right)$, and $a u b$ mutants $\left(a u b^{H N 2} / a u b^{Q C 42}\right)$ using the anti-AGO3 antibody revealed that the amount of AGO3 protein was severely reduced in $a u b$ mutant testes (Fig. 1A). The ago3 mRNA levels were also significantly affected in $a u b$ mutant testes (Fig. 1A, lower panel). In contrast, levels of AGO3 protein were not affected in $a u b$ mutant ovaries, suggesting that Aub is required for stabilizing ago3 mRNA and AGO3 protein in testes. Conversely, levels of Aub protein were not altered in either testes or ovaries of the ago3 mutants (Fig. 1A). Immunofluorescent staining of AGO3 in testes revealed that AGO3 was present in the cytoplasm of germline stem cells (GSC), gonialblasts, and spermatogonia, as was the case for Aub (Fig. 1B). However, AGO3 was below the level of detection in primary spermatocytes, where Aub is expressed. No expression of AGO3 or Aub was detected in somatic cells surrounding the gonialblasts and spermatogonia, or in the hub (Fig. 1B), where the strong expression of Piwi was observed (Cox et al. 2000; Saito et al. 2006). Both AGO3 and Aub accumulated in the nuage (Snee and Macdonald 2004; Brennecke et al. 2007), a ring around the cytoplasmic face of the nuclei in germline cells (Eddy 1975). Recent work has suggested the nuage to be a potential site for RISC-mediated post-transcriptional transposon silencing and piRNA biogenesis (Brennecke et al. 2007; Lim and Kai 2007; Li et al. 2009; Malone et al. 2009). In aub mutant testes, no nuage staining of AGO3 was observed. Instead, AGO3-positive large dots were occasionally observed in the cytoplasm, which was also the case for $a u b$ mutant ovaries (Malone et al. 2009). However, mutations in ago3 did not disrupt the localization of Aub to the nuage in the testes. These results show that Aub is required for AGO3 to be stabilized

B


FIGURE 1. AGO3 expression in fly testes. $(A)$ The expression levels of Aub and AGO3 were analyzed by Western blotting using anti-Aub and anti-AGO3 antibodies. The expression levels of AGO3 were severely reduced by loss of Aub expression in testes, while Aub was expressed in ago3 testes to the same extent as in wild-type $(y w)$ testes. The expression of ago 3 mRNA was reduced to almost $50 \%$ of the control level. The expression levels of aub and ago 3 mRNAs in $a u b$ and ago 3 mutant testes standardized on $a u b /+$ hetero or ago3/+ hetero line testes (red line) by qRT-PCR. This result showed that ago 3 mRNA was unstable in $a u b$ mutants. In contrast, such a severe reduction in AGO3 was not observed in $a u b$ mutant ovaries, indicating that the requirement for Aub in stabilizing AGO3 expression differs between the ovaries and the testes. (B) Fluorescent image of testes stained with anti-AGO3 and anti-Aub. Double-staining of fly testes [wild-type (wt) (top), aub (middle), ago3 (bottom)] with anti-Aub (red) and anti-AGO3 (green) antibodies. Aub and AGO3 expression was detected in germline stem cells (GSCs), gonialblast cells $(\mathrm{Gb})$, spermatogonia $(\mathrm{Sg})$, and spermatocytes $(\mathrm{Sc})$. (White triangle) The hub. Aub was expressed in primary spermatocytes, where AGO3 expression was below the limit of detection. Aub and AGO3 co-localize at the nuage, which is a perinuclear electron-dense structure in GSCs and gonialblasts. Aub localization at the nuage was not altered by loss of ago3 function; however, AGO3 did not accumulate at the nuage in aub mutants, indicating that AGO3 accumulation at the nuage depends on Aub. The AGO3-positive, large foci were observed near the nucleus in gonialblasts.

and to localize to the nuage in the testes. Thus, in the testes, the dependence of these PIWI proteins for their stability and localization to the nuage is different from that observed in the ovaries, where loss of AGO3 does not affect Aub protein levels and localization of AGO3 and 
Aub to the nuage is mutually interdependent ( $\mathrm{Li}$ et al. 2009; Malone et al. 2009).

\section{Analysis of piRNAs associated with AGO3 and Aub in fly testes by sequencing}

We previously performed a small-scale sequencing study to identify small RNAs associated with Aub, immunoprecipitated from testis lysate with an anti-Aub antibody (Nishida et al. 2007). To gain further insight into piRNA biogenesis in testes, we immunopurified AGO3 and Aub with specific antibodies from fly testes and performed a largescale sequencing to comprehensively examine their associated small RNAs. Both immunopurified AGO3 and Aub in testes (Fig. 2A) were associated with small RNAs 23-28 nt long (Fig. 2B).

To characterize the piRNAs associated with AGO3 and Aub, we generated 70,323 and 108,439 sequencing reads, respectively, for AGO3- and Aub-associated piRNAs. The list of piRNAs obtained is shown in Table 1 and Supplemental Table 1. piRNAs were mapped to the Drosophila genome and annotated (Fig. 2C). Fifty-four percent of the AGO3-associated piRNAs corresponded to transposons. In sharp contrast, only $7 \%$ of the Aub-associated piRNAs corresponded to transposons. The Aubassociated piRNAs corresponding to transposons mainly arose from antisense transcripts and showed a strong preference for $U$ at their $5^{\prime}$ ends, while the AGO3-associated transposon piRNAs were mainly derived from sense transcripts and showed a strong preference for $\mathrm{A}$ at the tenth nucleotide from the $5^{\prime}$ end (Fig. 3A,B). Among transposonderived piRNAs in Aub, $\sim 17 \%$ have ping-pong partner piRNAs, while $\sim 27 \%$ of transposon-derived piRNAs in AGO3 have ping-pong partner piRNAs (Supplemental Fig. 1). Thus, piRNAs corresponding to transposons in the testes show signatures of the ping-pong amplification cycle. These results suggest that piRNAs of transposon origin are produced by the amplification loop in testes as in ovaries. Curiously, we found that the expression levels of transposons in the testes were only slightly affected by the loss of $a u b$ or ago3 functions (Supplemental Fig. 2). This is in agreement

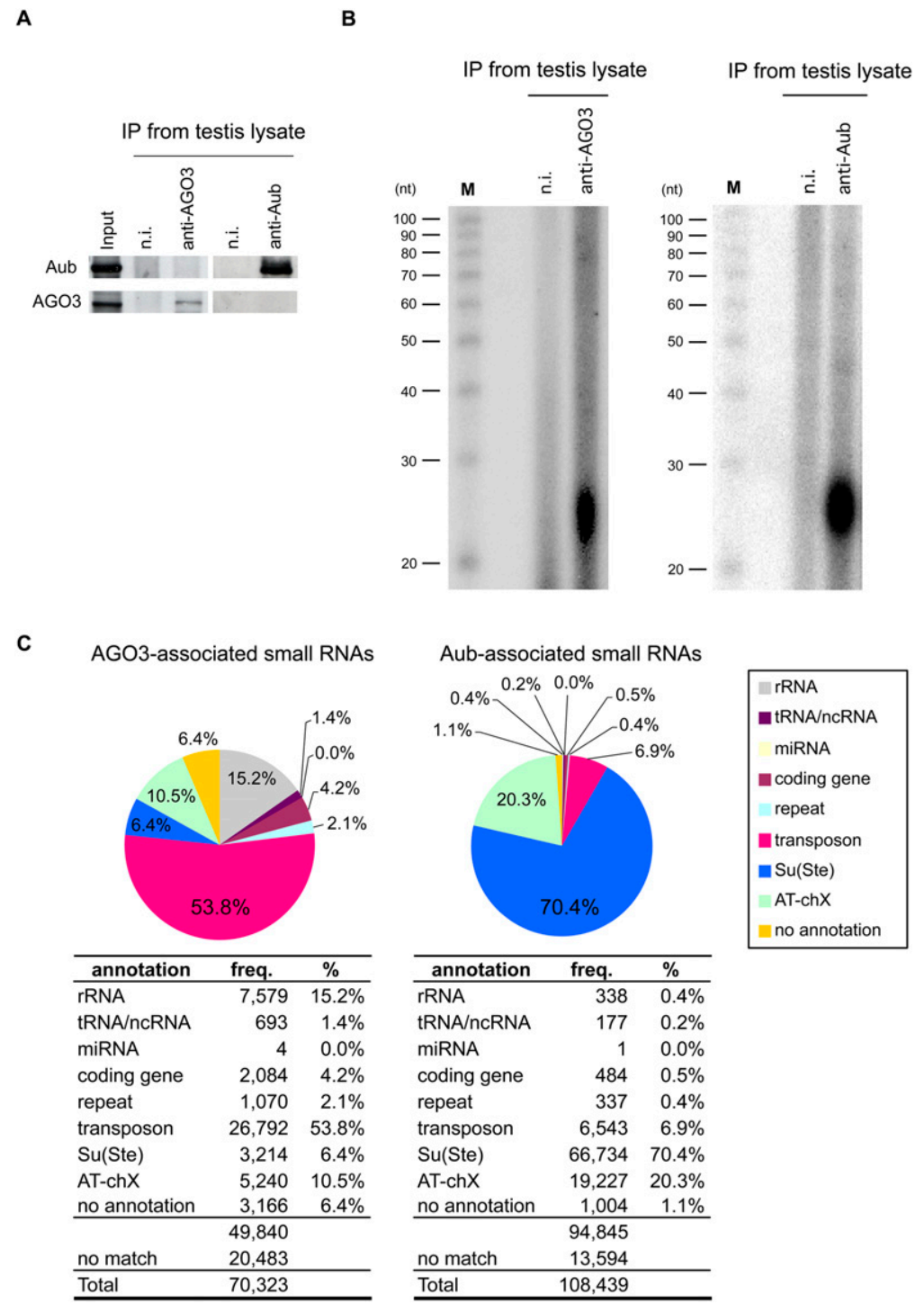

FIGURE 2. Analyses of small RNAs associated with AGO3 and Aub in testes. (A) Immunoprecipitation was performed from wild-type fly testes using anti-AGO3 and anti-Aub antibodies. (B) RNA molecules extracted from the immunoprecipitated complexes were visualized by ${ }^{32} \mathrm{P}$-ATP labeling on denaturing acrylamide gel. Small RNAs, 23-28 nt long, were observed associated with AGO3 and Aub in the testes. (C) Profiles of small RNAs associated with AGO3 and Aub in fly testes. The most abundant class of piRNAs associated with Aub was those derived from the Suppressor of Stellate $[\mathrm{Su}(\mathrm{Ste})]$ antisense transcripts. The second most abundant class of piRNAs associated with Aub was made up of those derived from an intergenic repetitive region on the X-chromosome, termed AT-chX. On the other hand, the majority of piRNAs associated with AGO3 were derived from transposons and other repetitive DNA elements (repeats) found in the genome. Su(Ste) piRNAs $(\sim 5 \%)$ as well as $A T$-chX piRNAs $(\sim 10 \%)$ were also present in the AGO3 small RNA library.

with findings that the testis expression of several retrotransposons was not significantly affected by $a u b$ mutations (Aravin et al. 2001), and these findings together raise the possibility that there exist unknown AGO3/Aub-independent mechanism(s) controlling transposon silencing in germline cells in the testes.

Although most transposon-derived piRNA species identified in the ovaries were sequenced only once (Brennecke 
TABLE 1. Top 50 small RNAs in AGO3 complexes in testes

\begin{tabular}{|c|c|c|c|c|c|c|}
\hline Sequence & $\begin{array}{c}\text { Read } \\
(\mathrm{AGO} 3)\end{array}$ & $\begin{array}{l}\text { Read } \\
\text { (Aub) }\end{array}$ & mismatch & $\begin{array}{c}\text { Annotation } \\
\text { (category) }\end{array}$ & $\begin{array}{l}\text { Annotation } \\
\text { (detail) }\end{array}$ & Strand \\
\hline UGUUUCAUCGUUAGACGGCUCGGGC & 2642 & 13,615 & 0 & AT-chX & AT-chX-B & Antisense \\
\hline AAAACCUGAAGAUGAUCCUGAGUCGC & 2229 & 119 & 0 & Transposon & Helena & Sense \\
\hline UGCUCUCGAAUGUAUGCCCGAUAU & 2069 & 17 & 0 & Transposon & R1-element & Antisense \\
\hline UCUCAUCGUCGUAGAACAAGCCCGA & 1670 & 43,207 & 0 & $\mathrm{Su}(\mathrm{Ste})$ & Su(Ste) & Antisense \\
\hline UGUUUCAUCGUUAGACGGCUCGGG & 875 & 3013 & 0 & AT-chX & AT-chX-B & Antisense \\
\hline AUGGCUCUCUCAGUCGCUUCCCGGGA & 857 & 3 & 0 & ncRNA & rRNA & Sense \\
\hline UGCUCUCGAAUGUAUGCCCGAUA & 729 & 4 & 0 & Transposon & R1-element & Antisense \\
\hline UGUUUCAUCGUUAGACGGCUCGGGCG & 693 & 91 & 0 & AT-chX & AT-chX-B & Antisense \\
\hline UGCUCUCGAAUGUAUGCCCGAUAUA & 693 & 8 & 0 & Transposon & R1-element & Antisense \\
\hline UGAGACUACAAACGGAUUGCGGACC & 606 & 0 & 0 & No annotation & & \\
\hline AGCUUCAUCCAAGCGAACUGUGCCCGA & 479 & 0 & 0 & Transposon & R1-element & Antisense \\
\hline ACGAGUAAUAUGAGCGCCCAGAGU & 441 & 0 & 0 & Transposon & opus & Sense \\
\hline UGCUCUCGAAUGUAUGCCCGAUAUAC & 374 & 2 & 0 & Transposon & R1-element & Antisense \\
\hline AAAACCUGAAGAUGAUCCUGAGUC & 366 & 24 & 0 & Transposon & Helena & Sense \\
\hline AAAACCUGAAGAUGAUCCUGAGUCG & 357 & 24 & 0 & Transposon & Helena & Sense \\
\hline UGUUUCAUCGUUAGACGGCUCGG & 308 & 134 & 0 & AT-chX & AT-chX-B & Antisense \\
\hline AAACCUGAAGAUGAUCCUGAGUCGC & 283 & 16 & 0 & Transposon & Helena & Sense \\
\hline CCCGAAAGCAGACGAGGUAACGUACC & 274 & 0 & 0 & Transposon & F-element & Sense \\
\hline AACUGAAUAAACGAAAUGGAUGACA & 255 & 0 & 0 & Transposon & invader4 & Sense \\
\hline ACGAGUAAUAUGAGCGCCCAGAGUGA & 241 & 0 & 0 & Transposon & opus & Sense \\
\hline AAAAACCUGAAGAUGAUCCUGAGUCGC & 241 & 17 & 1 & Transposon & Helena & Sense \\
\hline UUCAAGGUUACCCCAGGAUGCUGU & 238 & 80 & 0 & Repeat & $\operatorname{trf}$ & \\
\hline UACUUGUUCCCCGGAUAGUUUAGUUAC & 234 & 0 & 0 & ncRNA & rRNA & Sense \\
\hline GUAACUUCGGGAUAAGGAUUGGCUCU & 216 & 42 & 0 & ncRNA & rRNA & Sense \\
\hline ACAUUCAUAACCGAGCGACUGUUCAA & 188 & 0 & 0 & Transposon & GATE & Sense \\
\hline ACCGAAUUAAUCGCGAGGCUGCAAGA & 181 & 0 & 0 & Transposon & mdg3 & Sense \\
\hline UGUUUCAUCGUUAGACGGCUCGGGCGG & 175 & 0 & 0 & AT-chX & AT-chX-B & Antisense \\
\hline AACUGAAUAAACGAAAUGGAUGAC & 165 & 0 & 0 & Transposon & invader4 & Sense \\
\hline GCCGAACGUAGUCUCUCGAGAGCGA & 164 & 41 & 0 & Transposon & invader1 & Sense \\
\hline CAAACCGAUAUGCUAUCUUGGCUGA & 158 & 0 & 0 & Transposon & Doc & Sense \\
\hline UGGGAACACCGCGUGUUGUUGGCCU & 152 & 2 & 0 & ncRNA & 5SrRNA & Sense \\
\hline UCUCAUCGUCGUAGAACAAGCCCG & 151 & 6212 & 0 & $\mathrm{Su}(\mathrm{Ste})$ & Su(Ste) & Antisense \\
\hline ACCGAAUUAAUCGCGAGGCUGCAA & 147 & 0 & 0 & Transposon & mdg3 & Sense \\
\hline CAAGCUAGACUAUGUAUCCCCGGGUAA & 147 & 0 & 0 & Transposon & Doc & Sense \\
\hline AAAACCUGAAGAUGAUCCUGAGUCGCA & 143 & 41 & 0 & Transposon & Helena & Sense \\
\hline CCGAACUUGAGACCGCCCCUGGACGACC & 139 & 0 & 0 & $\mathrm{Su}(\mathrm{Ste})$ & $\mathrm{Su}(\mathrm{Ste})$ & Antisense \\
\hline AACUUCGGGAUAAGGAUUGGCUCU & 131 & 35 & 0 & ncRNA & rRNA & Antisense \\
\hline UACGACGAGAACGAGAUCAGCGCGA & 124 & 0 & 0 & Transposon & frogger & Antisense \\
\hline CAAACCAAUCCCAACCAAACCCGUCAA & 123 & 0 & 0 & Transposon & G2 & Sense \\
\hline UUCAAGGUUACCCCAGGAUGCUGUGC & 121 & 19 & 0 & Repeat & $\operatorname{trf}$ & \\
\hline UUUGCAAAAACGAGAGCGAUAGAGC & 113 & 0 & 0 & Transposon & micropia & Sense \\
\hline GCCAAUUACAUUAGACGAGACGGCCC & 111 & 3 & 0 & Transposon & opus & Sense \\
\hline GUGGCUUUGAGAGGCGCCGUAGUUA & 109 & 0 & 0 & No annotation & & \\
\hline AGCUUCAUCCAAGCGAACUGUGCCCG & 104 & 0 & 0 & Transposon & R1-element & Antisense \\
\hline ACCCUUGAUAAACGGUUGACUUUCG & 103 & 0 & 0 & Transposon & Rt1b & Sense \\
\hline UGAGAUAUGCUAGCAGACUGGAGA & 102 & 0 & 0 & Transposon & G2 & Sense \\
\hline UCGGCCCGAACCCUUCUGGUGUGU & 102 & 2 & 0 & Transposon & baggins & Sense \\
\hline AACUGAAUAAACGAAAUGGAUGACAC & 101 & 0 & 0 & Transposon & invader4 & Sense \\
\hline ACCCUUGAUAAACGGUUGACUUUC & 98 & 0 & 0 & Transposon & Rt1b & Sense \\
\hline UCGGCCCGAACCCUUCUGGUGUGUU & 96 & 1 & 0 & Transposon & baggins & Sense \\
\hline
\end{tabular}

Note that a large number of piRNAs with exactly the same sequences are present in both AGO3- and Aub-associated complexes.

et al. 2007; Li et al. 2009; Malone et al. 2009), we noted that piRNAs derived from some transposons including Helena, Opus, and Mgd3 elements in the testes, were sequenced multiple times (Table 1; Supplemental Table 1). For example, a piRNA derived from a sense strand of Helena was sequenced 4187 times in the AGO3 library and 314 times in the Aub library. These piRNAs with multiple reads tended to be derived from the sense strands of transposons (Fig. $3 \mathrm{~A})$. We found no antisense piRNAs that paired with these multiple-read sense piRNAs with signatures of an amplification cycle, namely, 1U/10A partners with a 10-nt, $5^{\prime}$-overlap. 
A

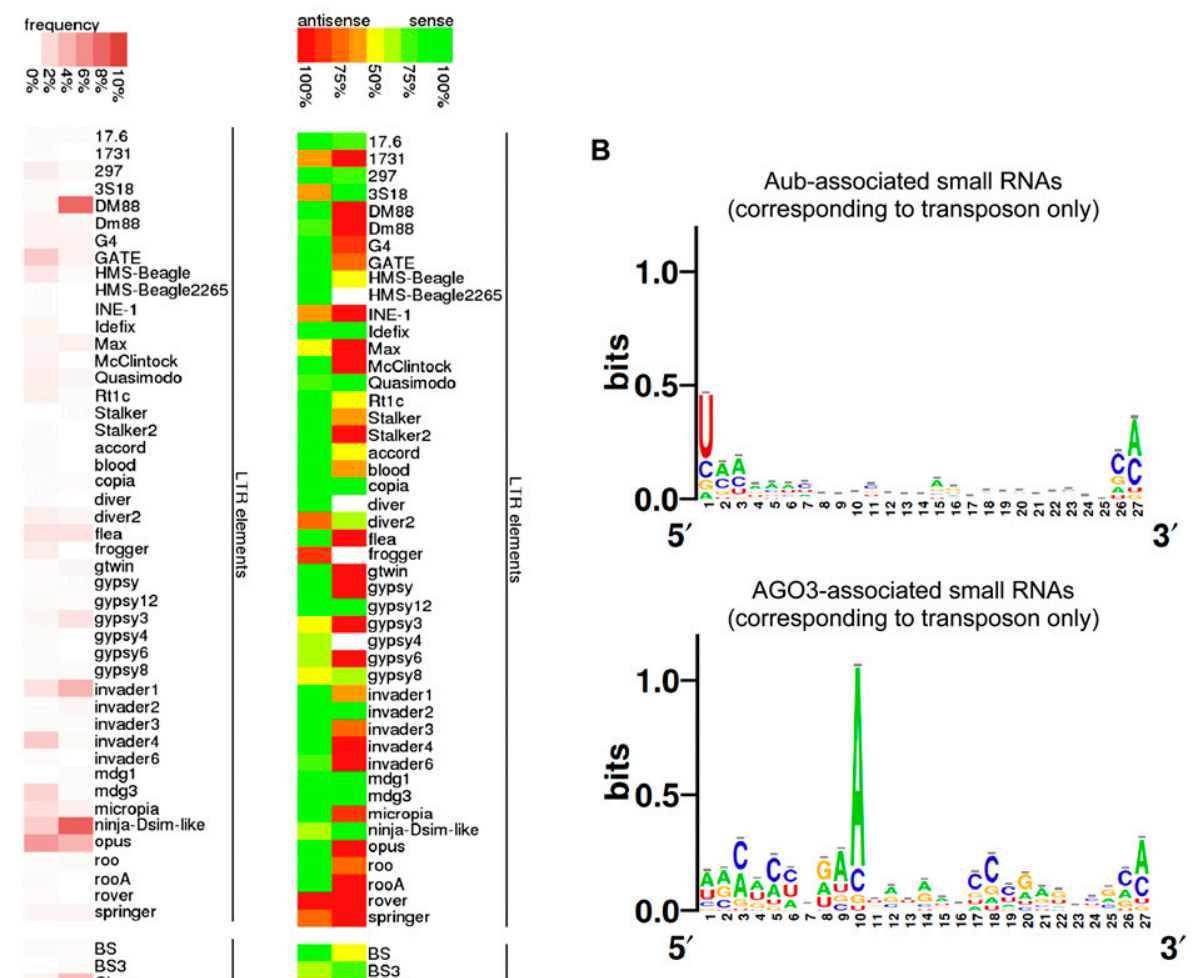

FIGURE 3. Bioinformatics analysis of piRNAs in testes. (A) The heat map (left panel) indicates the strand bias of cloned piRNAs with respect to canonical transposon sequences. Transposons are grouped into long terminal repeat (LTR), long interspersed nuclear (LINE), and inverted repeat (IR) elements. The color intensities indicate the degree of strand bias: (green) sense; (red) antisense; (yellow) unbiased. Aub-associated piRNAs mainly arose from antisense transcripts of transposons, and AGO3-associated piRNAs were mainly derived from sense transcripts. The cloning frequencies of individual transposons are also indicated as a heat map (right panel). (B) The basic composition of piRNAs from transposons indicates that Aub-associated piRNAs show a strong preference for $\mathrm{U}$ at their $5^{\prime}$ ends. On the other hand, AGO3-associated piRNAs contain predominantly A at the tenth nucleotide from the $5^{\prime}$ end. piRNAs corresponding to the $S u(S t e)$ and $A T$-chX loci were not included in this analysis.

\section{Su(Ste) piRNAs}

The most abundant class of Aub-associated piRNAs identified comprised those derived from $\mathrm{Su}(\mathrm{Ste})$ antisense tran- scripts (70.4\%) (Figs. 2C, 4; Supplemental Table 2). Of all the $S u(S t e)$ piRNAs associated with Aub, 65\% comprised only one piRNA, termed $S u(S t e)-4$ piRNA (Nishida et al. 2007). If up to two mismatches were permitted, $\sim 87 \%$ of 

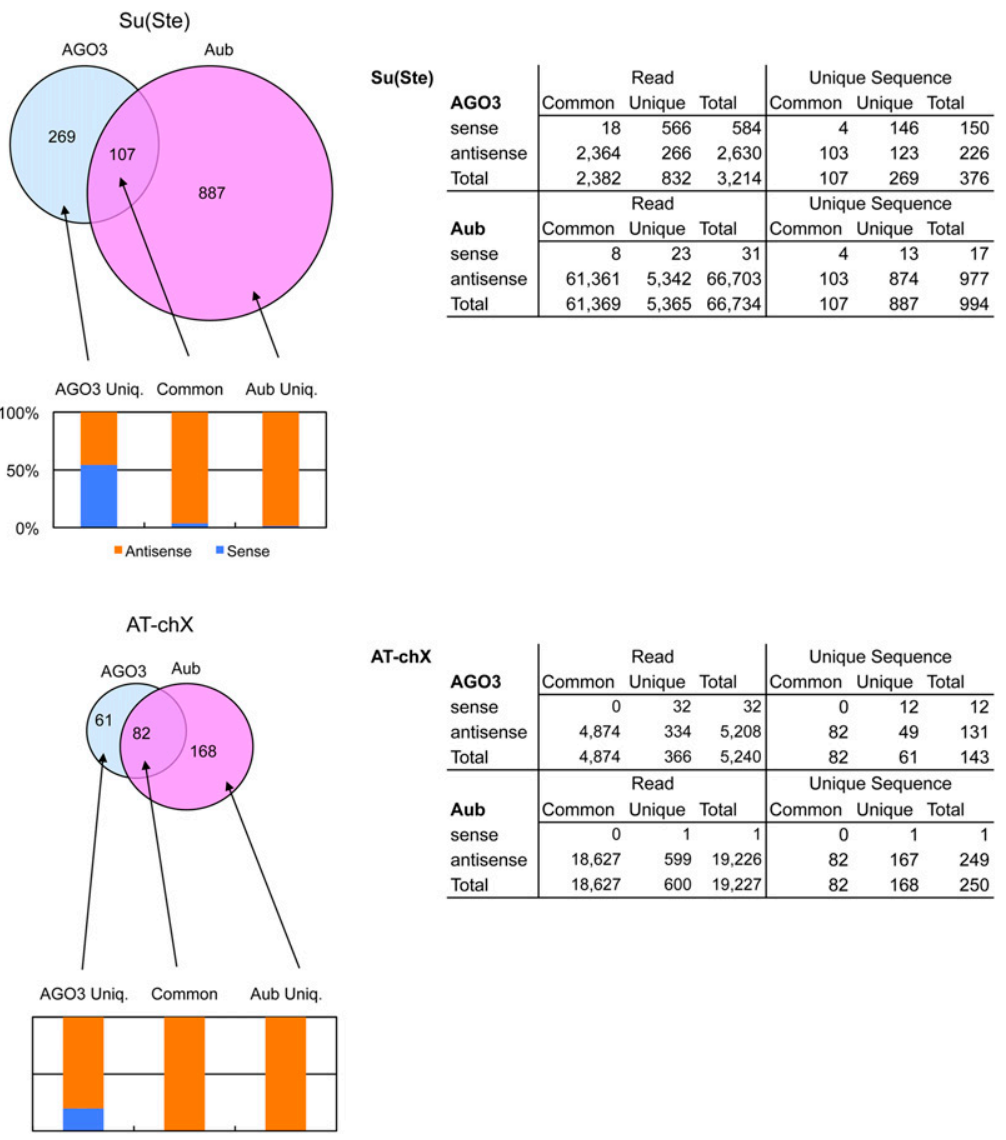

FIGURE 4. Classification of $S u(S t e)$ and $A T-c h X$ piRNAs loaded onto AGO3 and Aub. Aubassociated $S u(S t e)$ and $A T-c h X$ piRNAs were overwhelmingly antisense. A proportion of $\mathrm{Su}(\mathrm{Ste})$ and $A T$-chX piRNAs associated with AGO3 were sense-oriented. The classification of $\mathrm{Su}(\mathrm{Ste})$ and $A T-\operatorname{chX}$ piRNA sequences loaded onto both AGO3 and Aub, with the read numbers, is also listed below. The vast majority of $\mathrm{Su}(\mathrm{Ste})$ piRNAs loaded onto both AGO3 and Aub comprised those derived from $\mathrm{Su}(\mathrm{Ste})$ antisense transcripts, including the most abundant $S u(S t e)-4$ piRNA. Half of $S u(S t e)$ piRNA sequences loaded onto AGO3 alone (AGO3 Uniq.) comprised those derived from $S u(S t e)$ sense transcripts and only a portion of $A T$-chX sequences loaded onto Aub alone (Aub Uniq.) were all antisense. AT-chX piRNA sequences loaded onto both AGO3 and Aub (Common) comprised almost exclusively those derived from $A T$-ch $X$ antisense transcripts, including the most abundant $A T$-chX-1 piRNAs. piRNAs associated with AGO3 (AGO3 Uniq.) were sense-oriented. In contrast, $\mathrm{Su}(\mathrm{Ste})$ piRNA

$\sim 52 \%$ corresponded to $\mathrm{Su}(\mathrm{Ste})-4$ piRNA ( $\sim 67 \%$ if allowing up to two mismatches) and $\sim 85 \%$ were antisense-oriented (Supplemental Fig. 3). The majority of AGO3-associated $\mathrm{Su}$ (Ste) piRNA species were antisense-oriented and were also found among the Aub-associated Su(Ste) piRNAs (Supplemental Table 2). These results indicate that $\mathrm{Su}(\mathrm{Ste})$ piRNAs are mostly produced from antisense precursor molecules and that a large number of $\mathrm{Su}(\mathrm{Ste})$ piRNAs, mostly $\mathrm{Su}(\mathrm{Ste})-4$ piRNA and its variants, with exactly the same sequences, are loaded onto both AGO3 and Aub.

Among the AGO3-associated $\mathrm{Su}$ (Ste) piRNAs, $\sim 15 \%$ (584 out of 3214 reads) were sense-oriented (Fig. 4). To examine whether $\mathrm{Su}(\mathrm{Ste})$ piRNAs are produced in a ping-pong-dependent manner, we analyzed all the $S u(S t e)$ piRNAs bound to AGO3 and Aub to determine whether the first $10 \mathrm{nt}$ of $\mathrm{Su}(\mathrm{Ste})$ piRNAs in the sense orientation were complementary to the first $10 \mathrm{nt}$ of $\mathrm{Su}(\mathrm{Ste})$ piRNAs in the antisense orientation (Fig. 4). We found that only four Aub- and 11 AGO3associated sense $\mathrm{Su}(\mathrm{Ste})$ piRNAs have 78 Aub- and 38 AGO3-associated antisense $\mathrm{Su}(\mathrm{Ste})$ piRNA pairs showing signatures of an amplification cycle with $1 \mathrm{U} / 10 \mathrm{~A}$ partners with a 10 -nt, $5^{\prime}$-overlap (Supplemental Table 3). Although this suggests that the ping-pong cycle may still operate for the production of $\mathrm{Su}(\mathrm{Ste})$ piRNAs, albeit rarely, we were unable to find sense $S u(S t e)$ piRNAs that paired with $\mathrm{Su}(\mathrm{Ste})-4$ piRNA or its variants. We also failed to detect by Northern blotting the Aub-associated $S u(S t e)$ piRNAs $(58,055$ out of 66,734 reads) mapped to the $\mathrm{Su}(\mathrm{Ste})-4$ piRNA (Supplemental Table 2). The over-representation of the Su(Ste)-4 piRNA in the Aub library is consistent with our previous results obtained by analyzing a small Aub library that was independently produced and sequenced by a different method (Nishida et al. 2007). We also confirmed by Northern blot analysis that $S u(S t e)-4$ is a highly abundant piRNA in the testis (Supplemental Fig. 5A). This suggests that $\mathrm{Su}$ (Ste) piRNAs are not evenly produced from the precursor molecules, but they are produced from only very few hotspots (Supplemental Fig. 3). Su(Ste) piRNAs in the sense orientation corresponded to only 31 reads out of 66,734 reads among all the Aub-associated $\mathrm{Su}(\mathrm{Ste})$ piRNAs in this screening (Fig. 4). Among the AGO3-associated piRNAs in testes, $\sim 6 \%$ corresponded to $\mathrm{Su}(\mathrm{Ste}$ ) piRNAs (Fig. 2C), in which sense piRNAs that pair with $\mathrm{Su}(\mathrm{Ste})-4$ piRNA (Supplemental Fig. 5A). Taken together, these results suggest that the production of most of the $S u(S t e)$ piRNAs only through a ping-pong pathway is unlikely.

\section{AT-chX piRNAs}

A second large class of piRNAs associated with Aub in the testes was made up of those derived from a short repeated region, termed $A T-\operatorname{ch} X$, on chromosome $\mathrm{X}$ (Nishida et al. 2007). One of these piRNAs, termed $A T-\operatorname{ch} X-1$ (Nishida et al. 2007), showed strong complementarity to vas mRNA and suppresses the expression of VAS protein (Nishida et al. 2007). We analyzed AGO3- and Aub-associated AT-chX piRNAs and found that $10 \%$ and $20 \%$ of AGO3- and Aubassociated piRNAs, respectively, were derived from the 
AT-chX locus (Fig. 2C). These piRNAs were overwhelmingly antisense: Only 32 out of 5240 reads for AGO3-associated $A T$-chX piRNAs, and only one (one out of 19,227 reads) for Aub-associated $A T$ chX piRNAs were sense-oriented (Fig. 4; Supplemental Fig. 4). Only one pair (AT-chX-71 and $A T$-chX-71 sense) with ping-pong signatures was found among all these $A T$-chX piRNAs (Supplemental Fig. 4). Ninety-three percent of AGO3associated $A T$-chX piRNA species were also found among the Aub-associated $A T$-chX piRNAs (Fig. 4). If two mismatches were permitted, $\sim 91 \%$ of all Aub-associated $A T-\operatorname{chX}$ piRNAs, and $\sim 93 \%$ of all AGO3-associated $A T$-chX piRNAs, were mapped to $A T-\operatorname{ch} X-1$ and its variants (Supplemental Table 4). However, we failed to find sense piRNAs that pair with $A T-\operatorname{ch} X-1$ piRNA among Aub- and AGO3-associated AT-chX piRNAs. We confirmed by Northern blot analysis that the $A T-\operatorname{ch} X-1$ piRNA is a very abundant piRNA in the testis (Supplemental Fig. 5A). We also confirmed by Northern blotting the expression of $A T$-chX-71 piRNA that pairs with $A T$ chX-71 sense piRNA. However, we failed to detect $A T-\operatorname{ch} X-71$ sense piRNA and sense piRNAs that pair with $A T-\operatorname{ch} X-1$ piRNA (Supplemental Fig. 5A). Together, these results suggest that the production of piRNAs from the $A T-\operatorname{ch} X$ locus, particularly for the $A T-\operatorname{ch} X-1$ piRNA, through a ping-pong mechanism only is highly unlikely.

\section{Mutational analysis to define the genetic requirements for $\mathrm{Su}(\mathrm{Ste})$ and $A T$-ch $X$ piRNA production in testes}

It is known that both AGO3 and Aub are required to produce or stabilize $S u(S t e)$ piRNAs (Vagin et al. 2006; Li et al. 2009), suggesting mutual interdependence of the PIWI proteins for the biogenesis of $\mathrm{Su}(\mathrm{Ste})$ piRNAs. It is also known that AGO3 is required to produce or stabilize $A T-\operatorname{ch} X$ piRNAs (Li et al. 2009). We used Northern hybridization to examine $\mathrm{Su}(\mathrm{Ste})-4$ and $A T-\operatorname{ch} X-1$ piRNA production in ago3 and aub mutant testes (Fig. 5A). Neither $\mathrm{Su}(\mathrm{Ste})-4$ nor $A T$-chX-1 piRNAs were detected in aub mutant testes. A marked reduction in both piRNA species was observed in ago3 mutant testes. However, production of these piRNAs
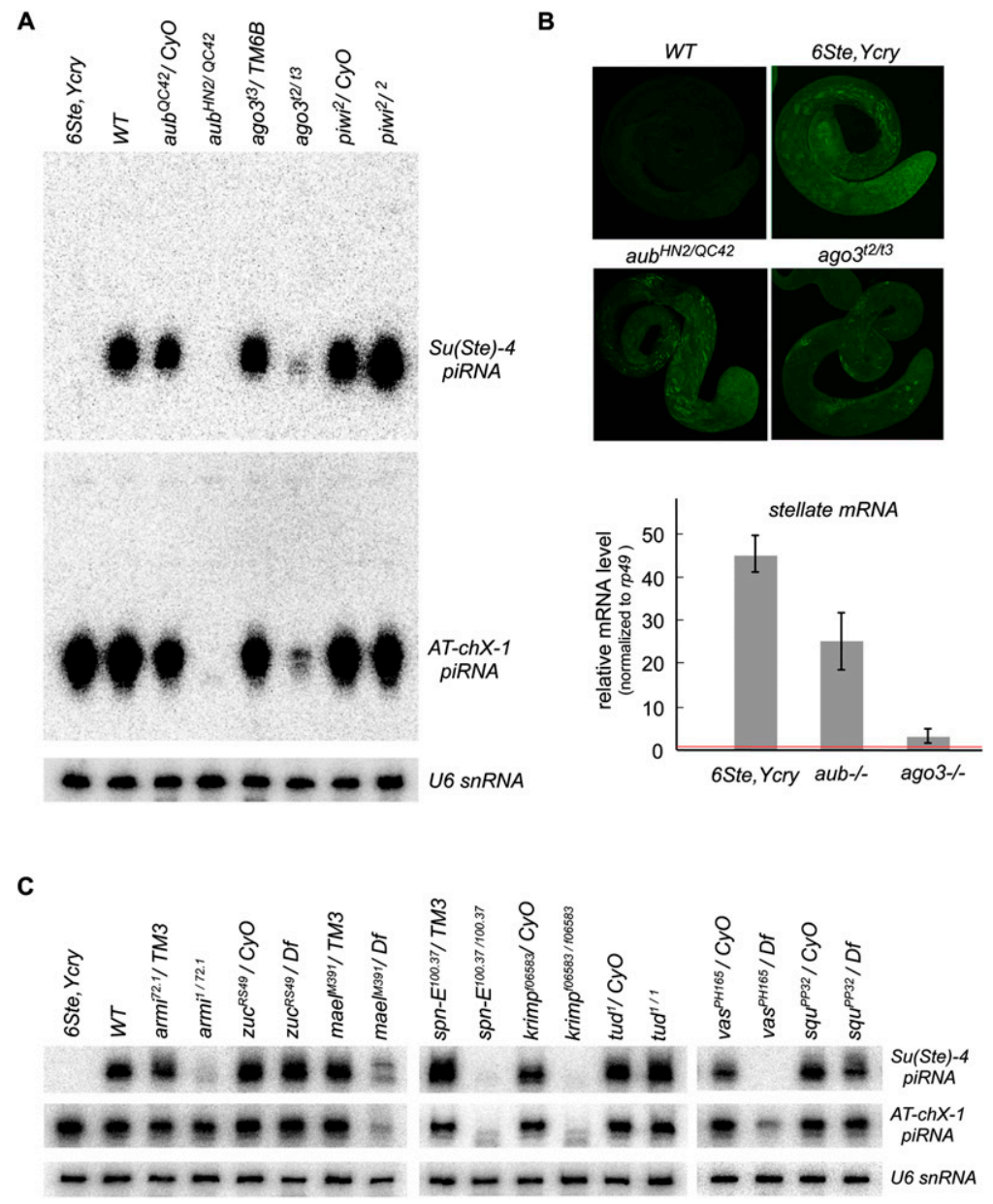

FIGURE 5. Genetic requirements for production of $S u(S t e)$ and At-chX piRNAs. (A) Accumulation of $S u(S t e)-4$ and $A T-c h X-1$ piRNAs in ago3 and $a u b$ mutant testes was analyzed by Northern blotting. The probe used was a DNA oligodeoxynucleotide containing a sequence complementary to $\mathrm{Su}(\mathrm{Ste})-4$ and $A T-c h X-1$. As expected, $\mathrm{Su}(\mathrm{Ste})-4$ piRNA was not detected in testes from males lacking the $S u(S t e)$ loci (6Ste, Ycry). In ago3 mutant testes, Su(Ste)-4 and AT$\operatorname{chX}-1$ piRNAs were detected only weakly, although in $a u b$ testes neither piRNA was detected. (B) Staining patterns of fly wild-type ( $y w)$, aub, ago3, and cry mutant testes with anti-Stellate antibody (upper panel). As expected, in the cry mutant (6Ste, Ycry) males, which have a deletion of the $\mathrm{Su}(\mathrm{Ste})$ locus, needle-shaped Stellate aggregation (crystals) were formed in primary spermatocytes. Stellate protein crystals were also observed in primary spermatocytes of $a u b$ testes, while ago3 testes contained far fewer crystals. The expression level of stellate mRNA was analyzed by qRT-PCR. The expression level of stellate mRNA in aub and ago3 mutant testes by qRT-PCR standardized to testes (red line) from wild type $(y w)$ or each hetero fly line $(a u b /+$, ago3/+) (lower panel). Loss of aub caused a dramatic increase in stellate mRNA expression, whereas loss of ago3 caused a slight increase in stellate mRNA expression. $(C)$ Northern analysis of the $S u(S t e)-4$ and $A T-c h X-1$ piRNAs in testes mutant for piRNA pathway genes. It should be noted that loss of function of $s p n-E$ and krimp resulted in a shift in the overall size of the population of $A T-\operatorname{ch} X-1$ piRNAs toward that of endo-siRNAs and miRNAs.

was not affected in piwi mutant testes, consistent with the fact that Piwi in the testes is expressed mostly in somatic cells, where AGO3 and Aub are not expressed (Nishida et al. 2007). These results suggest that both $\mathrm{Su}(\mathrm{Ste})-4$ and $A T-\operatorname{ch} X-1$ piRNAs are produced in germ cells by a mechanism requiring both AGO3 and Aub, even though these piRNAs have few or no signatures of the ping-pong cycle.

Previous genetic studies have shown that both ago3 and $a u b$ mutations cause the formation of Stellate crystals 
in the testes (Fig. 5B; Bozzetti et al. 1995; Aravin et al. 2004; Vagin et al. 2006; Li et al. 2009). We noted that, as reported (Li et al. 2009), Stellate protein crystals form in primary spermatocytes in ago3 testes but not as abundantly as in $a u b$ testes. We examined the accumulation of Stellate mRNA in these mutant testes and found that the level of Stellate mRNA was only slightly increased in ago3 testes, compared with that in $a u b$ testes (Fig. 5B). This may be accounted for by the low expression level of $\mathrm{AGO} 3$ protein in testes. The amount of AGO3 in testes was approximately 10 times lower than that of Aub in the testes (Supplemental Fig. 6). In addition, residual $S u(S t e)$ piRNAs in ago3 mutant testes may still be loaded onto Aub to form an RISC that silences Stellate mRNA, which may account for the weaker phenotype observed in ago3 mutant testes (Fig. 5A,B).

Mutations in a large number of genes affect piRNA production in Drosophila (Vagin et al. 2006; Chen et al. 2007; Lim and Kai 2007; Nishida et al. 2007; Pane et al. 2007; Klattenhoff and Theurkauf 2008; Li et al. 2009; Malone et al. 2009). To understand their involvement in the production of $S u(S t e)-4$ and $A T-\operatorname{ch} X-1$ piRNAs, we examined the molecular phenotypes of a series of eight mutants, including putative helicases, nucleases, and $\mathrm{Tu}-$ dor-domain proteins. We used Northern hybridization to examine $S u(S t e)-4$ and $A T-c h X-1$ piRNA production in these mutant testes. As shown in Figure 5C, both $\mathrm{Su}(\mathrm{Ste})-4$ and $A T-c h X-1$ piRNAs were strongly reduced in $s p n-E$ and krimp mutant testes. spn-E encodes a member of the DExH family of adenosine triphosphatases (ATPases) with a Tudor domain (Gillespie and Berg 1995), and mutations in this gene are known to impair Stellate silencing by eliminating $\mathrm{Su}(\mathrm{Ste})$ piRNAs (Aravin et al. 2004; Vagin et al. 2006). krimp encodes a Tudor-domain protein (Barbosa et al. 2007; Lim and Kai 2007). Both genes are critical for silencing of transposons and the accumulation of piRNAs in the Drosophila germline (Vagin et al. 2006; Lim and Kai 2007). Normal accumulation of both $\mathrm{Su}(\mathrm{Ste})-4$ and $A T-\operatorname{ch} X-1$ piRNAs also requires mael and vas. mael was identified as a genetic loss-of-function mutant, whose germline cells exhibited incorrect posterior localization of several transcripts (Clegg et al. 1997; Lim and Kai 2007). Mael has an MHG box and a domain homologous to DnaQ-H 3'-to-5' exonucleases (Zhang et al. 2008). vas is a germline-specific gene that encodes a DEAD-box RNA helicase involved in oogenesis (Lasko and Ashburner 1988; Styhler et al. 1998).

zucchini $(z u c)$ and squash (squ) were identified in a screen for female sterile mutations and cause dorso-ventral patterning defects (Schupbach and Wieschaus 1991; Pane et al. 2007). Both encode proteins with homology with nucleases. Mutations in squ slightly reduced the accumulation of Su(Ste)-4 piRNA and AT-chX-1 piRNA. However, the accumulation of neither piRNA was significantly affected in $z u c$ mutant testes.

$\mathrm{Su}$ (Ste)-4 piRNA was almost absent from armitage (armi) mutant testes, consistent with previous results (Vagin et al.
2006). Mutations in armi disrupt translational repression and localization of oskar mRNA and block RNA interference (RNAi) in Drosophila oocytes (Cook et al. 2004; Tomari et al. 2004). armi encodes a homolog of Arabidopsis SDE-3, an RNA helicase, which plays a role in posttranscriptional gene silencing triggered by transgenes and some viruses (Dalmay et al. 2001). The mammalian Armi homolog Mov10 also plays a role in siRNA-directed RNAi in cultured human cells (Meister et al. 2005). However, loss of Armi function had little impacts on AT-chX-1 piRNA levels.

We probed other piRNAs [Su(Ste)-6 and Su(Ste)-pair4] from the $\mathrm{Su}(\mathrm{Ste})$ locus on the Northern blots and found that the genetic requirements for their accumulation are almost identical to those for the $\mathrm{Su}(\mathrm{Ste})-4$ piRNA (Supplemental Fig. 5B,C). We also probed another piRNA (AT$\operatorname{chX}$-71) from the $A T$-ch $X$ locus on the Northern blots and found that, in contrast with $A T-\operatorname{ch} X-1$ piRNA, the accumulation of this piRNA appears dependent on armi, although modest, as compared with piRNAs from the $\mathrm{Su}(\mathrm{Ste})$ locus (Supplemental Fig. 5B,C).

Taken together, these results indicate that although production of both $S u(S t e)-4$ and $A T-c h X-1$ piRNAs requires several common genes, including ago3, aub, spn-E, krimp, and vas, which is similar to the requirements for the pingpong mechanism in the ovaries (Malone et al. 2009), the impacts of armi mutants on the operation of the piRNA pathway are variable in germ cells of fly testes.

\section{DISCUSSION}

The present study demonstrated that piRNAs in the germ cells of fly testes are produced not only by a ping-pong cycle but probably also by other mechanisms whose genetic requirements vary depending on the piRNA loci.

A surprisingly large portion of Aub-associated piRNAs in testes comprised only two piRNA species: $S u(S t e)-4$ and $A T$-chX-1. These piRNAs also comprise a large proportion of AGO3-associated piRNAs in testes. These piRNAs are not bound to Piwi that is expressed only in somatic cells in testes (Nishida et al. 2007). Therefore, these very abundant piRNAs are produced only in germ cells of the testes. In germ cells of the ovaries, $\mathrm{AGO} 3$ and Aub mostly engage in the ping-pong cycle to amplify piRNAs derived from transposons (Li et al. 2009; Malone et al. 2009). Although transposon-derived piRNAs that associate with AGO3 and Aub in testes show signatures of the ping-pong cycle, $\mathrm{Su}(\mathrm{Ste})$ and $A T$-chX piRNAs show very few signatures of the ping-pong cycle. The ping-pong cycle requires initiators to amplify piRNAs. piRNA populations maternally inherited by germline transmission serve as important initiators of the ping-pong cycle to amplify piRNAs in the ovaries (Blumenstiel and Hartl 2005; Brennecke et al. 2008). However, few or no $S u(S t e)$ piRNAs or AT-chX piRNAs are produced in the ovaries (Nishida et al. 2007). They are 
therefore not maternally passed on to the offspring to serve as inputs to the ping-pong cycle. Thus, these piRNAs cannot rely on maternally deposited piRNA populations to initiate the production in testes. In other words, the Drosophila testis must have evolved these sets of piRNA pathway genes to produce $S u(S t e)$ and $A T$-chX piRNAs. In ovaries, the primary piRNAs, whose production is mostly dependent on Piwi, have been proposed to be important initiators of the ping-pong cycle (Brennecke et al. 2007; Malone et al. 2009). Although Piwi is coexpressed with AGO3 and Aub in germ cells of the ovaries, its expression in testes is largely restricted to somatic cells where AGO3 and Aub are not expressed. Indeed, production of $\mathrm{Su}(\mathrm{Ste})-4$ and $A T-c h X-1$ piRNAs is Piwi-independent. Together, these findings suggest that production of $\mathrm{Su}(\mathrm{Ste})-4$ and $A T-\operatorname{chX}-1$ piRNAs through a ping-pong mechanism only is highly unlikely. However, it is still formally possible that, as suggested in Li et al. (2009), very few sense piRNAs might be sufficient to guide the processing of a large number of antisense piRNAs in a ping-pong manner.

How are these very abundant piRNAs produced in germ cells of the testes? Genetic analysis has revealed that in fly ovaries, ago3, aub, krimp, spn-E, and vas are required for the normal production of the ping-pong-dependent piRNAs derived from transposons in germ cells ( $\mathrm{Li}$ et al. 2009; Malone et al. 2009). However, only piwi and zuc mutations specifically decrease levels of piRNAs produced by the primary pathway in ovarian somatic cells (Malone et al. 2009; Saito et al. 2009). armi plays an important role in the pingpong mechanism (Malone et al. 2009). We demonstrated that in testes, ago3, aub, krimp, spn-E, and vas are required for the production of both $S u(S t e)-4$ and $A T-\operatorname{ch} X-1$ piRNAs in germ cells. This resembles the genetic requirements of the ping-pong cycle in the ovaries (Malone et al. 2009). However, these piRNAs in testes do not require piwi for their production. In addition, the $A T-\operatorname{ch} X-1$ piRNA accumulation does not require armi. These results therefore suggest that the $S u(S t e)-4$ and $A T-c h X-1$ piRNA pathways rely on different sets of genes.

The production of both $S u(S t e)-4$ and $A T-\operatorname{chX}-1$ piRNAs is dependent on both AGO3 and Aub. How does such interdependence between the two proteins occur? Mutations in aub severely impair the expression of AGO3 protein in testes. Thus, neither AGO3 nor Aub is sufficiently abundant for the loading of piRNAs in $a u b$ mutant testes. It is conceivable that, without the PIWI proteins, piRNAs are degraded. Supporting this is the observation that increased levels of Argonaute proteins in mammalian cells correlate with increased levels of mature miRNAs (Diederichs and Haber 2007). This effect depends on direct binding of the Argonaute proteins to the miRNA, suggesting that Argonaute proteins are limiting and serve to stabilize miRNAs. In sharp contrast, in ago3 mutant testes, the levels of Aub are not altered, but piRNA accumulation is markedly reduced. As $S u(S t e)-4$ and $A T-c h X-1$ piRNAs are mainly bound to Aub, it is difficult to explain how Aub could rely on AGO3 to stably produce these piRNAs in the testes. Because the accumulation of piRNA precursor- or intermediate-like molecules is not observed in ago3 and $a u b$ mutant testes (Fig. 5A), depletion of AGO3 and Aub may not substantially affect the processing of piRNA precursors. A plausible, although unsatisfying, model is that both proteins are required to form protein complexes that promote effective loading of piRNAs onto both AGO3 and Aub. Spn-E and Krimp are essential for the production of $S u(S t e)$ and $A T$-chX piRNAs, and both proteins contain Tudor domains that recognize and bind to symmetric dimethyl-arginine residues (sDMAs) on proteins (Côté and Richard 2005; Bedford and Clarke 2009). Recently it has been shown that PIWI proteins in fly ovaries contain sDMAs (Kirino et al. 2009; Nishida et al. 2009; Siomi et al. 2010) and that Tudor-domain-containing proteins such as Tudor interact with PIWI proteins specifically through their sDMA modifications (Nishida et al. 2009). In this context, it is tempting to speculate that AGO3 and Aub in fly testes may also contain sDMAs, which are required for the formation of functional complexes with Tudor-domain-containing proteins including spn-E and Krimp in the piRNA pathway. This model also implies that complexes containing AGO3 with sDMAs and complexes containing Aub with sDMAs are independently required for the piRNA pathway.

Our findings suggest that multiple pathways for piRNA biogenesis may exist in fly testes. More comprehensive bioinformatics, biochemical and genetic characterization of AGO3, Aub, and Piwi and their associated piRNAs in the Drosophila testis, should shed light on the molecular pathways of piRNA production.

\section{MATERIALS AND METHODS}

\section{Drosophila strains}

The yellow white $(y w)$ strain was used as the wild type. The strains bearing $a u b$ mutations, $a u b^{H N 2} c n b w / C y O$ and $a u b^{Q C 42} c n b w /$ $\mathrm{CyO}$, and ago3 mutations, ago $3^{\text {t2 }} / \mathrm{TM} 6 \mathrm{~B} \mathrm{~Tb}$ and ago $3^{\text {t3 }} / \mathrm{TM} 6 \mathrm{~B}$ $\mathrm{Tb}$ (Li et al. 2009), were provided by P. Zamore (University of Massachusetts, USA). The P[6Ste;Ste-lacZ]; Ycry Bs strains were provided by A. Aravin (Cold Spring Harbor Laboratory, NY, USA). Other mutant alleles and allelic combinations used in this work were $\mathrm{armi}^{1} / \mathrm{TM} 3 \mathrm{Sb} \mathrm{Ser}$ and $\mathrm{armi}^{72.1} / \mathrm{TM} 3 \mathrm{Sb} \mathrm{P}$ [hs-hid], PBac $\{W H\} k_{\text {rimp }} p^{f 06583} / C y O, p_{i w i}{ }^{2} / C y O$ (a kind gift from H. Lin, Yale Stem Cell Center), mael ${ }^{M 391} / T M 3 S b$ (a kind gift from T. Kai, Temasek Lifesciences Laboratory, Singapore) and Df(3L)BSC554/ TM6C Sb, spn- $E^{100.37}, e / T M 3, S b, P\left\{w^{+}, h s-h i d\right\}, s q u^{P P 32}$ cn bw /CyO and Df(2L)ED1109/SM6a, tud ${ }^{1}$ bw sp/CyO l(2)DTS513 ${ }^{1}$, vas $^{P H 165} / C y O$ (a kind gift from S. Kobayashi, NIBB, Okazaki), and $D f(2 L) A 267 \mathrm{bcn}$ $b w / C y O A d h, z u c^{R S 49} c n b w / C y O$ and $D f(2 L)$ Exel6031.

\section{Western blotting}

The anti-Aub monoclonal antibody (Nishida et al. 2007) was used at 1:1000 dilution, and the anti-AGO3 monoclonal antibody 
(Gunawardane et al. 2007) was used at 1:500 dilution. The antitubulin antibody was obtained from the Developmental Studies Hybridoma Bank and was used at 1:2000 dilution. Western blotting was performed as described previously (Miyoshi et al. 2005), with 20-40 $\mu \mathrm{g}$ of sample protein loaded on the gels.

\section{Immunofluorescence}

Testes and ovaries were dissected manually from adult flies in $1 \times$ phosphate-buffered saline (PBS). Immunostaining was performed following standard procedures. Anti-Aub was purified from culture supernatants of hybridoma cells using Thiophilic-Superflow Resion (BD Biosciences) and directly labeled using a HiLyte Fluor 555 Labeling Kit- $\mathrm{NH}_{2}$ (Dojindo Molecular Technologies). AntiAGO3 antibody (mouse polyclonal) was generated by using 289 amino acids at the $\mathrm{N}$ terminus of AGO3. Anti-AGO3 antibody was used at 1:5000 dilution. Alexa 488-conjugated anti-mouse IgG (Molecular Probes) was used as the secondary antibody to detect anti-AGO3 antibody. For Stellate staining, anti-Stellate antibody (a gift from P. Zamore) was used at 1:1000 dilution. Alexa 488-conjugated anti-rabbit IgG (Molecular Probes) was used as the secondary antibody to detect the anti-Stellate antibody. All images were collected using a Zeiss LSM510 laser scanning microscope.

\section{Immunoprecipitation}

Immunoprecipitation was performed using anti-Aub and antiAGO3 (polyclonal) antibodies immobilized on Dynabeads Protein $\mathrm{G}$ (Invitrogen). Immunoprecipitation buffer contained $50 \mathrm{mM}$ Tris- $\mathrm{HCl}$ ( $\mathrm{pH} 8.0$ ), $150 \mathrm{mM} \mathrm{NaCl}, 1 \mathrm{mM}$ dithiothreitol (DTT), $0.1 \%$ SDS, $1 \%$ Triton-X, $0.5 \%$ sodium deoxycholate, $2 \mu \mathrm{g} / \mathrm{mL}$ pepstatin, $2 \mu \mathrm{g} / \mathrm{mL}$ leupeptin, and $0.5 \%$ aprotinin. Approximately 1500 testes were used per immunoprecipitation. The reaction mixtures were rocked for $1 \mathrm{~h}$ at $4^{\circ} \mathrm{C}$, the bead fractions were washed five times with binding buffer, and the protein pools bound to the beads were eluted with SDS-PAGE sample buffer. After heating for $5 \mathrm{~min}$ to $95^{\circ} \mathrm{C}$, protein samples were run on SDS-PAGE gels and processed for Western blot analysis.

\section{Small RNA cloning and sequence analysis}

The cloning of small RNAs associated with Aub and AGO3 in the testes was carried out essentially as described (Saito et al. 2006) with minor modifications. After immunoprecipitation, total RNAs were isolated from the immunopurified complexes with phenol:chloroform and precipitated with ethanol. RNAs were dephosphorylated with calf intestinal phosphatase (CIP; NEB) and labeled with $\left[\gamma^{3}{ }^{32} \mathrm{P}\right]$ ATP with T4 polynucleotide kinase (TaKaRa) for visualization. For cloning of small RNAs isolated from the Aub and AGO3 complexes in the testes, we used adapters and primers [namely, the reverse transcription (RT) and PCR primers] as described in Saito et al. (2006). The adapters (MI-5' Linker and MI-3' Linker) and primers used for small RNAs associated with Aub and Piwi in the testes were included in the DynaExpress miRNA Cloning Kit (BioDynamics Laboratory). The sequences of each oligonucleotide were as follows:

MI-3' Linker: 5' -pCTGTAACTCGGGTCAATddC-3' (DNA)

MI-5' Linker: 5' -AUCGUCUCGGGAUGAAA-3' (RNA)

3' RT primer: 5'-ATTGACCCGAGTTACAG-3' (DNA)

5' Primer: 5'-ATCGTCTCGGGATGAAA-3' (DNA)
First-strand cDNA synthesis was performed with Stratascript RT (Stratagene) or Reverse Transcriptase (BioDynamics Laboratory). KOD plus (TOYOBO) or ExTaq polymerase (TaKaRa) was used as the polymerase. PCR products were cloned into the EcoRV site of the pBluescript SK+ vector and sequenced. Sequencing was performed on a GS FLX system (Roche), which produced 63,282 raw reads from the Aub library and 80,882 raw reads from the AGO3 library. We used a custom Perl script to remove $5^{\prime}-$ and 3 '-primer sequences found in the raw sequence data, and disjoined fused sequences that have multiple adapter sequences, which produce 108,439 short reads from the Aub library and 70,323 short reads from the AGO3 library. We mapped the resulting small RNA sequences to the genome sequence of Drosophila melanogaster (Apr. 2006 Assembly a.k.a dm3) by SeqMap (Jiang and Wong 2008) with two admissible mismatches, which resulted in 94,845 mapped sequences $(87.5 \%)$ in the Aub library and 49,840 mapped sequences (71\%) in the AGO3 library. The mapped sequences were annotated by comparing their genomic positions to track for repetitive elements (RepeatMasker, FlyBase natural transposable elements, and Tandem Repeat Finder), coding genes (FlyBase genes), non-coding genes (FlyBase non-coding genes), or the Functional RNA Database (Mituyama et al. 2008). Small RNA sequences were deposited in the Gene Expression Omnibus (www.ncbi.nlm.nih.gov/geo/) under the accession number SRP003750.

\section{Northern blotting}

Total RNA from fly testes was isolated using ISOGEN (Nippon Gene). Northern blotting was performed as reported previously (Saito et al. 2006) with minor modifications. Five micrograms of total RNA from each sample was separated on $12 \%$ acrylamidedenaturing gels and transferred onto Hybond-N membrane (Amersham Pharmacia) in distilled water and 1-ethyl-3 [3dimethylaminopropyl]carbodiimide hydrochloride cross-linking reactions for $2 \mathrm{~h}$ at $60^{\circ} \mathrm{C}$ to enhance detection of small RNA molecules (Pall and Hamilton 2008). After cross-linking, hybridization was performed at $42^{\circ} \mathrm{C}$ in $0.2 \mathrm{M}$ sodium phosphate ( $\mathrm{pH} 7.2$ ), $7 \%$ SDS, and $1 \mathrm{mM}$ EDTA with end-labeled antisense oligodeoxynucleotide, and washed at $42^{\circ} \mathrm{C}$ in $2 \times$ saline sodium citrate and $0.1 \%$ SDS. The probes used for detecting $\mathrm{Su}(\mathrm{Ste})-4$ piRNAs, $A T$ chX-1 piRNAs, and U6 snRNAs are listed in Supplemental Table 5.

\section{RT-PCR}

Quantitative RT-PCR (qRT-PCR) was performed as reported previously (Saito et al. 2009). One microgram of total RNA from each sample was used to reverse-transcribe target sequences using a Transcriptor First Strand cDNA Synthesis Kit (Roche) according to the manufacturer's instructions. The resulting cDNAs were amplified with a LightCycler 480 SYBR Green I Master (Roche). The primers used are shown in Supplemental Table 5.

\section{SUPPLEMENTAL MATERIAL}

Supplemental material can be found at http://www.rnajournal.org.

\section{ACKNOWLEDGMENTS}

We thank P. Zamore, A. Aravin, H. Lin, S. Kobayashi, and T. Kai for fly stocks and antibodies. We also thank S. Inagaki and 
H. Ishizu for technical advice, and Y. Iyoda for collecting fly testes. We thank members of the Siomi laboratory for discussion and comments on the manuscript. This work was supported by the Ministry of Education, Culture, Sports, Science and Technology of Japan (MEXT) grants to H.S. M.C.S. is supported by CREST (Core Research for Evolutional Science and Technology) from JST (Japan Science and Technology Agency) and the New Energy and Industrial Technology Development Organization (NEDO).

Received May 17, 2010; accepted September 16, 2010.

\section{REFERENCES}

Aravin AA, Naumova NM, Tulin AV, Vagin VV, Rozovsky YM, Gvozdev VA. 2001. Double-stranded RNA-mediated silencing of genomic tandem repeats and transposable elements in the D. melanogaster germline. Curr Biol 11: 1017-1027.

Aravin AA, Klenov MS, Vagin VV, Bantignies F, Cavalli G, Gvozdev VA. 2004. Dissection of a natural RNA silencing process in the Drosophila melanogaster germ line. Mol Cell Biol 24: 67426750.

Aravin AA, Hannon GJ, Brennecke J. 2007. The Piwi-piRNA pathway provides an adaptive defense in the transposon arms race. Science 318: $761-764$.

Balakireva MD, Shevelyov YY, Nurminsky DI, Livak KJ, Gvozdev VA. 1992. Structural organization and diversification of Y-linked sequences comprising $\mathrm{Su}(\mathrm{Ste})$ genes in Drosophila melanogaster. Nucleic Acids Res 20: 3731-3736.

Barbosa V, Kimm N, Lehmann R. 2007. A maternal screen for genes regulating Drosophila oocyte polarity uncovers new steps in meiotic progression. Genetics 176: 1967-1977.

Bedford MT, Clarke SG. 2009. Protein arginine methylation in mammals: Who, what, and why. Mol Cell 33: 1-13.

Blumenstiel JP, Hartl DL. 2005. Evidence for maternally transmitted small interfering RNA in the repression of transposition in Drosophila virilis. Proc Natl Acad Sci 102: 15965-15970.

Bozzetti MP, Massari S, Finelli P, Meggio F, Pinna LA, Boldyreff B, Issinger OG, Palumbo G, Ciriaco C, Bonaccorsi S, et al. 1995. The Ste locus, a component of the parasitic cry-Ste system of Drosophila melanogaster, encodes a protein that forms crystals in primary spermatocytes and mimics properties of the $\beta$ subunit of casein kinase 2. Proc Natl Acad Sci 92: 6067-6071.

Brennecke J, Aravin AA, Stark A, Dus M, Kellis M, Sachidanandam R, Hannon GJ. 2007. Discrete small RNA-generating loci as master regulators of transposon activity in Drosophila. Cell 128: 10891103.

Brennecke J, Malone CD, Aravin AA, Sachidanandam R, Stark A, Hannon GJ. 2008. An epigenetic role for maternally inherited piRNAs in transposon silencing. Science 322: 1387-1392.

Chen Y, Pane A, Schüpbach T. 2007. Cutoff and aubergine mutations result in retrotransposon upregulation and checkpoint activation in Drosophila. Curr Biol 17: 637-642.

Clegg NJ, Frost DM, Larkin MK, Subrahmanyan L, Bryant Z, Ruohola-Baker H. 1997. maelstrom is required for an early step in the establishment of Drosophila oocyte polarity: Posterior localization of grk mRNA. Development 124: 4661-4671.

Cook HA, Koppetsch BS, Wu J, Theurkauf WE. 2004. The Drosophila SDE3 homolog armitage is required for oskar mRNA silencing and embryonic axis specification. Cell 116: 817-829.

Côté J, Richard S. 2005. Tudor domains bind symmetrical dimethylated arginines. J Biol Chem 280: 28476-28483.

Cox DN, Chao A, Lin H. 2000. piwi encodes a nucleoplasmic factor whose activity modulates the number and division rate of germline stem cells. Development 127: 503-514.

Czech B, Malone CD, Zhou R, Stark A, Schlingeheyde C, Dus M, Perrimon N, Kellis M, Wohlschlegel JA, Sachidanandam R, et al.
2008. An endogenous small interfering RNA pathway in Drosophila. Nature 453: 798-802.

Dalmay T, Horsefield R, Braunstein TH, Baulcombe DC. 2001. SDE3 encodes an RNA helicase required for post-transcriptional gene silencing in Arabidopsis. EMBO J 20: 2069-2078.

Diederichs S, Haber DA. 2007. Dual role for argonautes in microRNA processing and posttranscriptional regulation of microRNA expression. Cell 131: 1097-1108.

Eddy EM. 1975. Germ plasm and the differentiation of the germ cell line. Int Rev Cytol 43: 229-280.

Ghildiyal M, Zamore PD. 2009. Small silencing RNAs: an expanding universe. Nat Rev Genet 10: 94-108.

Ghildiyal M, Seitz H, Horwich MD, Li C, Du T, Lee S, Xu J, Kittler EL, Zapp ML, Weng Z, et al. 2008. Endogenous siRNAs derived from transposons and mRNAs in Drosophila somatic cells. Science 320: 1077-1081.

Gillespie DE, Berg CA. 1995. Homeless is required for RNA localization in Drosophila oogenesis and encodes a new member of the $\mathrm{DE}-\mathrm{H}$ family of RNA-dependent ATPases. Genes Dev 9: 2495-2508.

Girard A, Hannon GJ. 2008. Conserved themes in small-RNA-mediated transposon control. Trends Cell Biol 18: 136-148.

Grimson A, Srivastava M, Fahey B, Woodcroft BJ, Chiang HR, King N, Degnan BM, Rokhsar DS, Bartel DP. 2008. Early origins and evolution of microRNAs and Piwi-interacting RNAs in animals. Nature 455: 1193-1197.

Gunawardane LS, Saito K, Nishida KM, Miyoshi K, Kawamura Y, Nagami T, Siomi H, Siomi MC. 2007. A slicer-mediated mechanism for repeat-associated siRNA 5' end formation in Drosophila. Science 315: 1587-1590.

Harris AN, Macdonald PM. 2001. aubergine encodes a Drosophila polar granule component required for pole cell formation and related to eIF2C. Development 128: 2823-2832.

Houwing S, Kamminga LM, Berezikov E, Cronembold D, Girard A, van den Elst $\mathrm{H}$, Filippov DV, Blaser H, Raz E, Moens CB, et al. 2007. A role for Piwi and piRNAs in germ cell maintenance and transposon silencing in Zebrafish. Cell 129: 69-82.

Jiang H, Wong WH. 2008. SeqMap: mapping massive amount of oligonucleotides to the genome. Bioinformatics 24: 2395-2396.

Kawamura Y, Saito K, Kin T, Ono Y, Asai K, Sunohara T, Okada TN, Siomi MC, Siomi H. 2008. Drosophila endogenous small RNAs bind to Argonaute 2 in somatic cells. Nature 453: 793-797.

Kawaoka S, Hayashi N, Suzuki Y, Abe H, Sugano S, Tomari Y, Shimada T, Katsuma S. 2009. The Bombyx ovary-derived cell line endogenously expresses PIWI/PIWI-interacting RNA complexes. RNA 15: 1258-1264.

Kim VN, Han J, Siomi MC. 2009. Biogenesis of small RNAs in animals. Nat Rev Mol Cell Biol 10: 126-139.

Kirino Y, Kim N, de Planell-Saguer M, Khandros E, Chiorean S, Klein PS, Rigoutsos I, Jongens TA, Mourelatos Z. 2009. Arginine methylation of Piwi proteins catalysed by dPRMT5 is required for Ago3 and Aub stability. Nat Cell Biol 11: 652-658.

Klattenhoff C, Theurkauf W. 2008. Biogenesis and germline functions of piRNAs. Development 135: 3-9.

Kotelnikov RN, Klenov MS, Rozovsky YM, Olenina LV, Kibanov MV, Gvozdev VA. 2009. Peculiarities of piRNA-mediated post-transcriptional silencing of Stellate repeats in testes of Drosophila melanogaster. Nucleic Acids Res 37: 3254-3263.

Lasko PF, Ashburner M. 1988. The product of the Drosophila gene vasa is very similar to eukaryotic initiation factor-4A. Nature 335: 611-617.

Li C, Vagin VV, Lee S, Xu J, Ma S, Xi H, Seitz H, Horwich MD, Syrzycka M, Honda BM, et al. 2009. Collapse of germline piRNAs in the absence of Argonaute3 reveals somatic piRNAs in flies. Cell 137: 509-521.

Lim AK, Kai T. 2007. Unique germ-line organelle, nuage, functions to repress selfish genetic elements in Drosophila melanogaster. Proc Natl Acad Sci 104: 6714-6719.

Malone CD, Brennecke J, Dus M, Stark A, McCombie WR, Sachidanandam R, Hannon GJ. 2009. Specialized piRNA pathways 
act in germline and somatic tissues of the Drosophila ovary. Cell 137: 522-535.

Megosh HB, Cox DN, Campbell C, Lin H. 2006. The role of PIWI and the miRNA machinery in Drosophila germline determination. Curr Biol 16: 1884-1894.

Meister G, Landthaler M, Peters L, Chen PY, Urlaub H, Lührmann R, Tuschl T. 2005. Identification of novel Argonaute-associated proteins. Curr Biol 15: 2149-2155.

Mituyama T, Yamada K, Hattori E, Okida H, Ono Y, Terai G, Yoshizawa A, Komori T, Asai K. 2008. The Functional RNA Database 3.0: databases to support mining and annotation of functional RNAs. Nucleic Acids Res 37: D89-D92.

Miyoshi K, Tsukumo H, Nagami T, Siomi H, Siomi MC. 2005. Slicer function of Drosophila Argonautes and its involvement in RISC formation. Genes Dev 19: 2837-2848.

Nishida KM, Saito K, Mori T, Kawamura Y, Nagami-Okada T, Inagaki S, Siomi H, Siomi MC. 2007. Gene silencing mechanisms mediated by Aubergine piRNA complexes in Drosophila male gonad. RNA 13: 1911-1922.

Nishida KM, Okada TN, Kawamura T, Mituyama T, Kawamura Y, Inagaki S, Huang H, Chen D, Kodama T, Siomi H, et al. 2009. Functional involvement of Tudor and dPRMT5 in the piRNA processing pathway in Drosophila germlines. EMBO J 28: 3820-3831.

Okamura K, Chung WJ, Ruby JG, Guo H, Bartel DP, Lai EC. 2008. The Drosophila hairpin RNA pathway generates endogenous short interfering RNAs. Nature 453: 803-806.

Pall GS, Hamilton AJ. 2008. Improved Northern blot method for enhanced detection of small RNA. Nat Protoc 3: 1077-1084.

Pane A, Wehr K, Schüpbach T. 2007. zucchini and squash encode two putative nucleases required for rasiRNA production in the Drosophila germline. Dev Cell 12: 851-862.

Pélisson A, Song SU, Prud'homme N, Smith PA, Bucheton A, Corces VG. 1994. Gypsy transposition correlates with the production of a retroviral envelope-like protein under the tissuespecific control of the Drosophila flamenco gene. EMBO $J$ 13: $4401-4411$.

Saito K, Nishida KM, Mori T, Kawamura Y, Miyoshi K, Nagami T, Siomi H, Siomi MC. 2006. Specific association of Piwi with rasiRNAs derived from retrotransposon and heterochromatic regions in the Drosophila genome. Genes Dev 20: 2214-2222.
Saito K, Inagaki S, Mituyama T, Kawamura Y, Ono Y, Sakota E, Kotani H, Asai K, Siomi H, Siomi MC. 2009. A regulatory circuit for piwi by the large Maf gene traffic jam in Drosophila. Nature 461: $1296-1299$.

Schüpbach T, Wieschaus E. 1991. Female sterile mutations on the second chromosome of Drosophila melanogaster. II. Mutations blocking oogenesis or altering egg morphology. Genetics 129: 1119-1136.

Siomi MC, Kuramochi-Miyagawa S. 2009. RNA silencing in germlines-exquisite collaboration of Argonaute proteins with small RNAs for germline survival. Curr Opin Cell Biol 21: 426-434.

Siomi H, Siomi MC. 2008. Interactions between transposable elements and Argonautes have (probably) been shaping the Drosophila genome throughout evolution. Curr Opin Genet Dev 18: 181-187.

Siomi H, Siomi MC. 2009. On the road to reading the RNAinterference code. Nature 457: 396-404.

Siomi MC, Mannen T, Siomi H. 2010. How does the Royal Family of Tudor rule the PIWI-interacting RNA pathway? Genes Dev 24: 636-646.

Snee MJ, Macdonald PM. 2004. Live imaging of nuage and polar granules: evidence against a precursor-product relationship and a novel role for Oskar in stabilization of polar granule components. J Cell Sci 117: 2109-2120.

Styhler S, Nakamura A, Swan A, Suter B, Lasko P. 1998. vasa is required for GURKEN accumulation in the oocyte, and is involved in oocyte differentiation and germline cyst development. Development 125: 1569-1578.

Tomari Y, Du T, Haley B, Schwarz DS, Bennett R, Cook HA, Koppetsch BS, Theurkauf WE, Zamore PD. 2004. RISC assembly defects in the Drosophila RNAi mutant armitage. Cell 116: 831-841.

Vagin VV, Sigova A, Li C, Seitz H, Gvozdev V, Zamore PD. 2006. A distinct small RNA pathway silences selfish genetic elements in the germline. Science 313: 320-324.

Williams RW, Rubin GM. 2002. ARGONAUTE1 is required for efficient RNA interference in Drosophila embryos. Proc Natl Acad Sci 99: 6889-6894.

Zhang D, Xiong H, Shan J, Xia X, Trudeau VL. 2008. Functional insight into Maelstrom in the germline piRNA pathway: a unique domain homologous to the DnaQ-H 3'-5' exonuclease, its lineagespecific expansion/loss and evolutionarily active site switch. Biol Direct 3: 48. doi: 10.1186/1745-6150-3-48. 

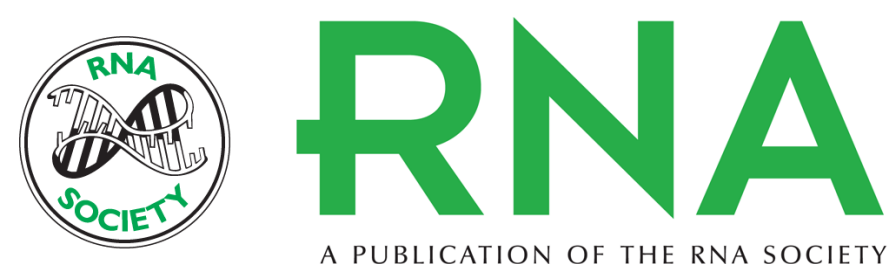

A PUBLICATION OF THE RNA SOCIETY

\section{Biogenesis pathways of piRNAs loaded onto AGO3 in the Drosophila testis}

Akihiro Nagao, Toutai Mituyama, Haidong Huang, et al.

RNA 2010 16: 2503-2515 originally published online October 27, 2010

Access the most recent version at doi:10.1261/rna.2270710

\section{Supplemental http://rnajournal.cshlp.org/content/suppl/2010/10/18/rna.2270710.DC1 \\ Material}

References This article cites 61 articles, 24 of which can be accessed free at:

http://rnajournal.cshlp.org/content/16/12/2503.full.html\#ref-list-1

\section{License}

Email Alerting Receive free email alerts when new articles cite this article - sign up in the box at the Service top right corner of the article or click here.

To subscribe to RNA go to:

http://rnajournal.cshlp.org/subscriptions 